\title{
Serum Asymmetric Dimethylarginine Concentrations in Two Models of Experimentally Induced Hypertension
}

\author{
M. Khazaei, ${ }^{1}$ M. Zarei, ${ }^{2}$ and N. Esmaeil ${ }^{3}$ \\ ${ }^{1}$ Department of Physiology, Isfahan University of Medical Sciences, Isfahan, Iran \\ ${ }^{2}$ Department of Physiology, Hamadan University of Medical Sciences, Hamadan, Iran \\ ${ }^{3}$ Department of Immunology, Isfahan University of Medical Sciences, Isfahan, Iran
}

Correspondence should be addressed to M. Khazaei; khazaei@med.mui.ac.ir

Received 13 November 2012; Accepted 2 December 2012

Academic Editors: M. Frick, K. Kotani, R. S. Padwal, and G. L. Schwartz

Copyright (c) 2013 M. Khazaei et al. This is an open access article distributed under the Creative Commons Attribution License, which permits unrestricted use, distribution, and reproduction in any medium, provided the original work is properly cited.

\begin{abstract}
The aim of this study was to evaluate the effect of two models of hypertension on serum ADMA concentrations and whether the changes of serum ADMA concentrations are reversible by removing the cause of hypertension. Materials and Methods. 48 male wistar rats were randomly assigned into four groups: control, deoxycorticosterone acetate salt (DOCA-Salt), sham, and two kidneys one clip (2K1C). After 12 weeks, in half of the animals ( $n=6$ each group), serums were taken and direct blood pressure was measured. Then, DOCA injection was withdrawn and the animals received tap water and, in 2K1C group, renal clips were removed. After 12 weeks, direct blood pressure was measured and blood samples were taken. Results. Serum ADMA concentration in DOCA-Salt group was slightly higher than control, although it was not statistically significant. In $2 \mathrm{~K} 1 \mathrm{C}$ hypertensive group, serum ADMA concentration was significantly elevated compared to sham group $(P<0.05)$. Unclipping and reversal of hypertension returned serum ADMA level to sham group. There was a weak positive correlation between systolic blood pressure and serum ADMA concentration. Conclusion. increased serum ADMA concentration during hypertension is dependent on the model of hypertension, and removing the cause of hypertension could reduce it
\end{abstract}

\section{Introduction}

Endothelial dysfunction is a major risk factor for cardiovascular disease which is accompanied with reduced nitric oxide (NO) bioavailability [1]. NO has several vascular protective effects including vasodilation, inhibition of platelet aggregartion, smooth muscle cell proliferation, and leukocyte adhesion [2]. Studies indicated that reduced bioavailability of NO by NO synthase inhibitor or increased NO degradation causes endothelial dysfunction [3]. Asymmetric dimethylarginine (ADMA) is an endogenous inhibitor of NO synthase and influence NO production [4]. ADMA is produced by protein arginine methyltransferase enzyme (PRMT) and competes with L-arginine, NO precursor, for all the three isoforms of NO synthase [5].

As an NO synthase inhibitor, ADMA has an important role in vascular homeostasis. There is a correlation between ADMA levels and cardiovascular morbidity and mortality [6, 7]. ADMA is associated by cardiovascular risk factors such as atherosclerosis, hypertension, and diabetes [8]. Elevated level of ADMA has been demonstrated in hyperlipidemia, diabetes mellitus, and peripheral artery disease [9]; however, there is a controversy on the effect of hypertension on serum ADMA concentration. Some clinical studies reported a relationship between hypertension and high plasma ADMA concentration $[1,10,11]$, while other studies have not shown this association $[12,13]$. On the other hand, little is known about the effect of reversal of hypertension without using drug on serum ADMA concentration. The purpose of this study was to determine the effect of two models of hypertension on serum ADMA concentration and whether the changes of ADMA level is reversible by removing the cause of hypertension.

\section{Materials and Methods}

2.1. Animals. The experiments were conducted in 48 male wistar rats, age and weight matched housed in a temperaturecontrolled animal room with a 12-hour light/dark cycle with 
TABLE 1: Systolic, diastolic, and mean arterial pressure (SBP, DBP, and MAP, resp.) in experimental groups. ${ }^{*} P<0.05$ compared to sham. ${ }^{\#} P<0.05$ compared to control.

\begin{tabular}{lcccc}
\hline & Subgroups & SBP $(\mathrm{mmHg})$ & DBP $(\mathrm{mmHg})$ & $\mathrm{MAP}(\mathrm{mmHg})$ \\
\hline \multirow{3}{*}{ K1C } & Preclipping & $105.65 \pm 2.65$ & $75.42 \pm 3.66$ & $82.24 \pm 1.31$ \\
& Postclipping & $165.84 \pm 3.79^{*}$ & $102.41 \pm 2.59^{*}$ & $126.72 \pm 3.77^{*}$ \\
\hline \multirow{3}{*}{ Sham } & Unclipping & $118.40 \pm 4.42$ & $81.22 \pm 3.77$ & $86.22 \pm 1.91$ \\
& Preclipping & $104.16 \pm 3.96$ & $71.33 \pm 2.33$ & $80.02 \pm 1.11$ \\
& Postclipping & $103.72 \pm 2.46$ & $72.98 \pm 5.33$ & $81.22 \pm 1.35$ \\
DOCA & Unclipping & $112.97 \pm 4.56$ & $75.34 \pm 4.76$ & $82.44 \pm 1.59$ \\
& Before experiment & $93.30 \pm 3.29$ & $80.04 \pm 1.02$ & $85.07 \pm 0.92$ \\
& DOCA & $151.50 \pm 2.18^{\#}$ & $105.90 \pm 4.32^{\#}$ & \\
Control & DOCA withdrawal & $94.72 \pm 4.6$ & $83.68 \pm 1.92$ & $84.52 \pm 2.51^{\#}$ \\
& Before experiment & $96.51 \pm 4.2$ & $82.34 \pm 3.76$ & $86.32 \pm 1.04$ \\
\hline
\end{tabular}

free access to rodent chow and tap water. The Ethical Committee of Isfahan University of Medical Sciences approved the study. After one week of habituation to the animal facility, the animals were randomly assigned into four groups: control, deoxycorticosterone acetate salt (DOCA-Salt), sham, and two kidneys one clip (2K1C) ( $n=12$ each).

\subsection{Induction of Hypertension and Reversal of Hypertension}

2.2.1. $2 \mathrm{~K} 1 \mathrm{C}$ Model of Hypertension. The animals were randomly divided into hypertensive $(n=12)$ and shamoperated groups $(n=12)$. For induction of hypertension, the animals were anaesthetized with intraperitoneally injection of ketamine hydrochloride $(75 \mathrm{mg} / \mathrm{kg})$ and xylazine $(7.5 \mathrm{mg} / \mathrm{kg})$. Left kidneys were exposed via flank incision and a silver clip (internal gap: $0.20 \mathrm{~mm}$ ) was put around the renal artery [14]. In sham-clipped group, the same procedure was done without using silver clip. After closing the wound, the animals were returned to the animal facilities. Blood pressure was measured twice a week using tail cuff method (ADInstruments, Australia). After 12 weeks, half of the animals in each group $(n=6$ each) were anaesthetized. Direct blood pressure was measured via a catheter (PE-50) inserted into the carotid artery. Blood samples were taken for subsequent determination of plasma renin activity (PRA) and serum ADMA concentrations. To investigate the effect of reversal of hypertension, the rest of the animals were anaesthetized ( $n=6$ each). Left renal arteries were exposed and the silver clips gently removed without damaging renal artery or causing bleeding (unclipping group). In shamunclipped group, the same procedure was done without removing the clip. Blood pressure was measured via tail cuff method every week. After 12 weeks, direct blood pressure was measured and blood samples were taken.

2.2.2. DOCA-Salt Model of Hypertension. The animals were uninephrectomized via left flank incision. After recovery, hypertensive group received subcutaneously injection of DOCA $(30 \mathrm{mg} / \mathrm{kg})$, twice a week and $\mathrm{NaCl} 1 \%$ solution instead of tap water [15]. Control group received solvent of DOCA with tap water. Blood pressure was measured every week using tail cuff pressure. After 12 weeks, in half of the animals, direct blood pressure was measured and blood samples were taken. For reversal of hypertension, DOCA injection was withdrawn and the animals received tap water instead of $\mathrm{NaCl} 1 \%$ solution. After 12 weeks, direct blood pressure was measured and blood samples were taken.

2.3. Blood Samples. Blood samples were taken and centrifuged at $3000 \mathrm{rpm}$ for 20 minutes. Plasma and serums were stored at $-70^{\circ} \mathrm{C}$ for further analysis.

2.4. PRA Measurement. Plasma renin activity (PRA) was measured in $2 \mathrm{~K} 1 \mathrm{C}$ and sham groups using radioimmunoassay kit (Renctk P2721, DiaSorin Biomedica Diagnostic RIA kit, Italy). PRA was calculated as nanogram angiotensin I generated $/ \mathrm{mL} / \mathrm{h}$. The PRA assay sensitivity was $0.13 \mathrm{ng} / \mathrm{mL}$.

2.5. Serum ADMA Measurement. Serum ADMA concentrations were measured by the competitive ADMA Elisa kit (DLD diagnostika, Germany). The minimum sensitivity of the assay was $0.05 \mu \mathrm{mol} / \mathrm{L}$.

2.6. Statistical Analysis. Data are reported as mean \pm SE. SPSS 16 software was used for analysis of data. One-way ANOVA using Tukey's post-hoc test was used for comparison of data between groups. Bivariate correlations were calculated using Pearson's correlation coefficient. $P$ less than 0.05 was considered statistically significant.

\section{Results}

3.1. Blood Pressure. As illustrated in Table 1, the animals in hypertensive groups (2K1C and DOCA-Salt) had significantly higher blood pressure compared to sham or control groups $P<0.05)$. Unclipping in $2 \mathrm{~K} 1 \mathrm{C}$ group and withdrawal of DOCA-Salt reduced blood pressure which returned to sham and control levels (Table 1). 


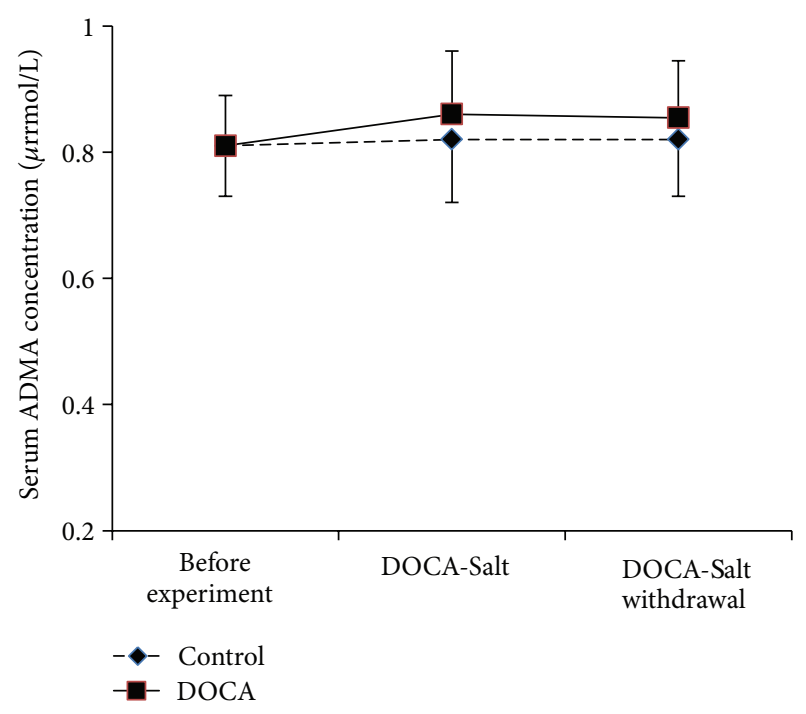

(a)

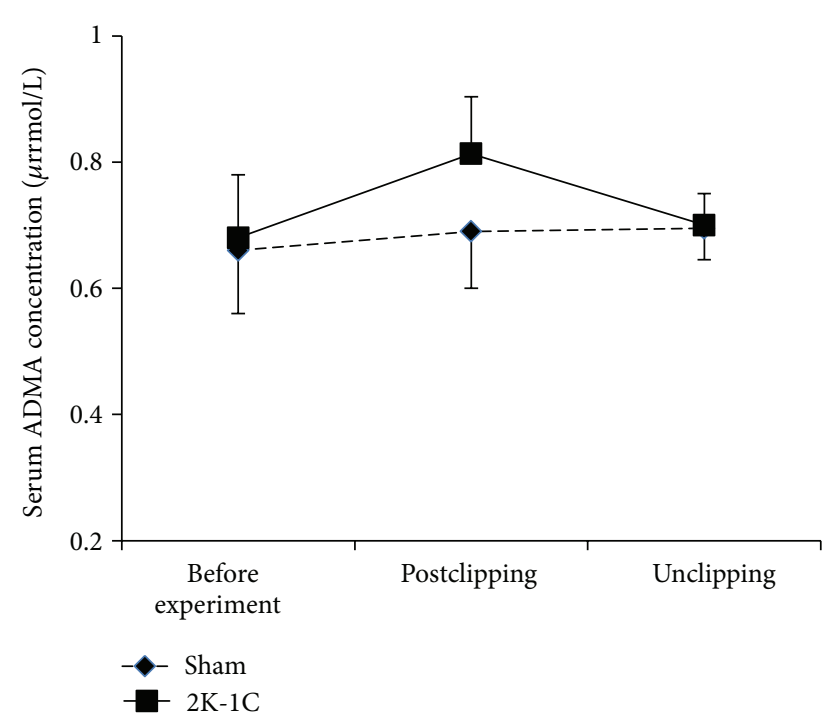

(b)

FIGURE 1: Changes of serum ADMA concentrations before and after induction of hypertension and after blood pressure reduction in DOCASalt (a) and 2K1C (b) hypertensive rats.

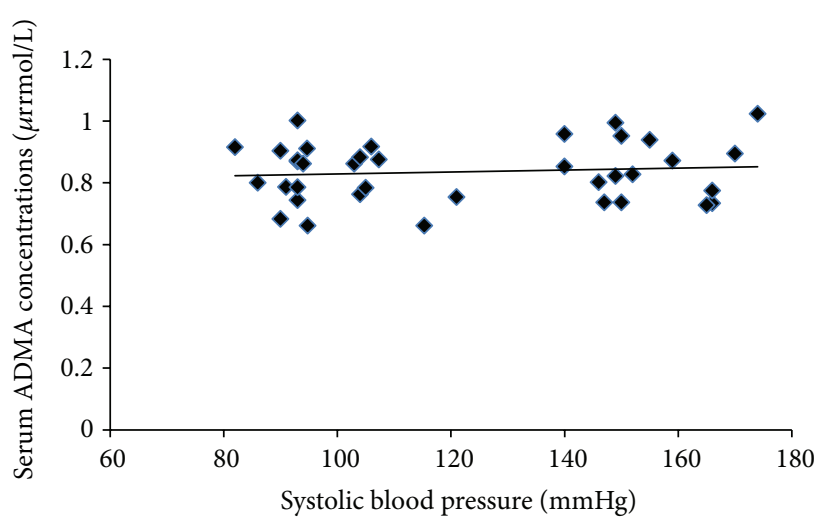

FIGURE 2: Correlation between serum ADMA concentration and systolic blood pressure $(r=0.15)$.

3.2. PRA Measurement. PRA levels were measured in 2K1C hypertensive rats. Results showed that PRA level was significantly higher in $2 \mathrm{~K} 1 \mathrm{C}$ compared to sham group $(5.90 \pm 0.20$ versus $3.10 \pm 0.13 \mathrm{ng} / \mathrm{mL} / \mathrm{h} ; P<0.05)$ and reduced after unclipping $(3.16 \pm 0.14$ versus $5.90 \pm 0.20 \mathrm{ng} / \mathrm{mL} / \mathrm{h} ; P<$ $0.05)$.

3.3. Serum ADMA Concentration. Serum ADMA concentrations in DOCA-Salt hypertensive group were higher than control, although it was not statistically significant. Reversal of hypertension did not change serum ADMA concentration. In $2 \mathrm{~K} 1 \mathrm{C}$ hypertensive group, serum ADMA concentration was significantly elevated compare to sham group $(P<0.05)$. Unclipping and reversal of hypertension returned serum ADMA levels to sham group (Figure 1).
3.4. Correlation between SBP and Serum ADMA Concentration. In the correlation analysis, there was a weak positive correlation between serum ADMA concentration and systolic blood pressure $(r=0.15)$ (Figure 2).

\section{Discussion}

Epidemiological and clinical studies demonstrated that ADMA can be considered as a strong cardiovascular risk factor and predict future cardiovascular events $[6,7,12]$. ADMA inhibits NO production which causes endothelial dysfunction [3]. Elevated ADMA has been demonstrated in several cardiovascular risk factors; however, there is a controversy on the effect of hypertension on serum ADMA concentration.

In this study, we found that, in DOCA-Salt hypertensive animals, serum ADMA concentration slightly increased while in 2K1C model, it significantly increased and returned to baseline level after unclipping. In a recent study, it is reported that in patients with metabolic syndrome, ADMA levels did not correlate with blood pressure [16]. Chirions et al. also found an association between ADMA and carotid atherosclerosis without correlation with blood pressure [17]. In contrast, several studies found a correlation between ADMA and blood pressure in patients with essential hypertension $[1,10,11]$. It is demonstrated that hypertensive subjects had significantly higher ADMA and L-arginine plasma concentrations and elevated ADMA is inversely related to endothelial function [1].

The clinical significance of ADMA has been reviewed [18]. The adverse effect of elevated ADMA is mediated by its effect on NO synthase inhibition which reduces NO production and leads to endothelial dysfunction [3]. 
In the present study, we found a slight increase in serum ADMA concentration in DOCA-Salt hypertensive animals. Although it was not statistically significant, it should be considered that a small change in the plasma level of ADMA has a large effect on the intracellular level of ADMA and is sufficient to alter NO production [19].

The reason of high plasma ADMA level during hypertension is unknown. Increased shear stress due to high blood pressure may increase ADMA synthesis [20]. High plasma ADMA may also be related to reduced catabolic rate and renal clearance [21]. It is demonstrated that when hypertension is associated with renal disease, increased ADMA level is observed [22]. Sasser et al. showed that elevated blood pressure in a model of angiotensin-II-induced hypertension in the absence of renal injury does not increase plasma ADMA level; however, in presence of kidney damage and in sustained hypertension for 6 weeks, plasma ADMA level increases and contributes to NO deficiency [22]. Reduced DDAH activity in renal cortex also may contribute to increased plasma ADMA concentration [22]. In the present study, we did not evaluate renal function and this is the limitation of this study.

It was shown that lung is the main source of circulating ADMA. In an in vitro study, incubation of endothelial cells with ang II for $24 \mathrm{~h}$ increased PRMT expression and increased ADMA level [23]. Thus, increased PRMT expression in the lung can contribute to the elevated plasma ADMA in 2K1C hypertensive animals. Another explanation for elevated serum ADMA concentration in $2 \mathrm{~K} 1 \mathrm{C}$ animals is that ADMA level may increase secondary to ang-II-induced oxidative stress. It is indicated that elevated level of ADMA may partly relate to development of oxidative stress with development of hypertension $[24,25]$.

Previous studies indicated that ACE inhibitor drugs such as perindopril or enalapril and AT1 receptor antagonists such as losartan or eprosartan reduced serum ADMA concentration in patients with essential hypertension [26, 27]. However, the main difference between the present study and those studies is that, in this study, we did not use any drug for blood pressure reduction. A recent study demonstrated that plasma ADMA level was significantly increased in patients with essential hypertension and positively related to SBP level [28]. However, in this study we observed a weak positive correlation between SBP and serum ADMA concentration.

In conclusion, it seems that increased serum ADMA concentration during hypertension is dependent on the type of hypertension, and removing the cause of hypertension could reduce it.

\section{Acknowledgment}

The authors thank the Vice Chancellor of Isfahan University of Medical Sciences for their financial support (Grant no. 287267).

\section{References}

[1] F. Perticone, A. Sciacqua, R. Maio et al., "Asymmetric dimethylarginine, L-arginine, and endothelial dysfunction in essential hypertension," Journal of the American College of Cardiology, vol. 46, no. 3, pp. 518-523, 2005.

[2] A. Cengel and A. Sahinarslan, "Nitric oxide and cardiovascular system," Anadolu Kardiyoloji Dergisi, vol. 6, no. 4, pp. 364-368, 2006.

[3] J. P. Cooke, "Does ADMA cause endothelial dysfunction?" Arteriosclerosis, Thrombosis, and Vascular Biology, vol. 20, no. 9, pp. 2032-2037, 2000.

[4] T. Teerlink, "Measurement of asymmetric dimethylarginine in plasma: methodological considerations and clinical relevance," Clinical Chemistry and Laboratory Medicine, vol. 43, no. 10, pp. 1130-1138, 2005.

[5] P. Vallance and J. Leiper, "Cardiovascular biology of the asymmetric dimethylarginine:dimethylarginine dimethylaminohydrolase pathway," Arteriosclerosis, Thrombosis, and Vascular Biology, vol. 24, no. 6, pp. 1023-1030, 2004.

[6] R. H. Böger, "Asymmetric dimethylarginine (ADMA) and cardiovascular disease: insights from prospective clinical trials," Vascular Medicine, vol. 10, no. 1, pp. S19-S25, 2005.

[7] J. Wang, A. S. Sim, X. L. Wang, C. Salonikas, D. Naidoo, and D. E. L. Wilcken, "Relations between plasma asymmetric dimethylarginine (ADMA) and risk factors for coronary disease," Atherosclerosis, vol. 184, no. 2, pp. 383-388, 2006.

[8] R. H. Böger, "The emerging role of asymmetric dimethylarginine as a novel cardiovascular risk factor," Cardiovascular Research, vol. 59, no. 4, pp. 824-833, 2003.

[9] K. Krzyzanowska, F. Mittermayer, M. Wolzt, and G. Schernthaner, "ADMA, cardiovascular disease and diabetes," Diabetes Research and Clinical Practice, vol. 82, no. 2, pp. S122-S126, 2008.

[10] A. Surdacki, M. Nowicki, J. Sandmann et al., "Reduced urinary excretion of nitric oxide metabolites and increased plasma levels of asymmetric dimethylarginine in men with essential hypertension," Journal of Cardiovascular Pharmacology, vol. 33, no. 4, pp. 652-658, 1999.

[11] D. Wang, S. Strandgaard, J. Iversen, and C. S. Wilcox, "Asymmetric dimethylarginine, oxidative stress, and vascular nitric oxide synthase in essential hypertension," American Journal of Physiology, vol. 296, no. 2, pp. R195-R200, 2009.

[12] R. Schnabel, S. Blankenberg, E. Lubos et al., "Asymmetric dimethylarginine and the risk of cardiovascular events and death in patients with coronary artery disease: results from the AtheroGene Study," Circulation Research, vol. 97, no. 5, pp. e53-59, 2005.

[13] A. Meinitzer, U. Seelhorst, B. Wellnitz et al., "Asymmetrical dimethylarginine independently predicts total and cardiovascular mortality in individuals with angiographic coronary artery disease (The Ludwigshafen Risk and Cardiovascular Health Study)," Clinical Chemistry, vol. 53, no. 2, pp. 273-283, 2007.

[14] F. Diekmann, R. Zart, C. Thone-Reineke, C. Bauer, H. H. Neumayer, and B. Hocher, "Regulation of the renal endothelin system in the two-kidney, one clip renal hypertensive rat," Journal of Cardiovascular Pharmacology, vol. 36, no. 5, pp. S191-S194, 2000.

[15] C. Veeramani, B. Aristatle, G. Pushpavalli, and K. V. Pugalendi, "Antihypertensive efficacy of Melothria maderaspatana leaf extract on sham-operated and uninephrectomized DOCA-salt hypertensive rats," Journal of Basic and Clinical Physiology and Pharmacology, vol. 21, no. 1, pp. 27-41, 2010. 
[16] I. Palomo, A. Contreras, L. M. Alarcón et al., "Elevated concentration of asymmetric dimethylarginine (ADMA) in individuals with metabolic syndrome," Nitric Oxide, vol. 24, no. 4, pp. 224-228, 2011.

[17] J. A. Chirinos, R. David, J. A. Bralley et al., "Endogenous nitric oxide synthase inhibitors, arterial hemodynamics, and subclinical vascular disease: the PREVENCION study," Hypertension, vol. 52, no. 6, pp. 1051-1059, 2008.

[18] M. P. Siroen, T. Teerlink, R. J. Nijveldt, H. A. Prins, M. C. Richir, and P. A. van Leeuwen, "The clinical significance of asymmetric dimethylarginine," Annual Review of Nutrition, vol. 26, pp. 203-228, 2006.

[19] A. J. Cardounel, H. Cui, A. Samouilov et al., "Evidence for the pathophysiological role of endogenous methylarginines in regulation of endothelial no production and vascular function," Journal of Biological Chemistry, vol. 282, no. 2, pp. 879-887, 2007.

[20] M. G. Modena, L. Bonetti, F. Coppi, F. Bursi, and R. Rossi, "Prognostic role of reversible endothelial dysfunction in hypertensive postmenopausal women," Journal of the American College of Cardiology, vol. 40, no. 3, pp. 505-510, 2002.

[21] J. M. Leiper, J. Santa Maria, A. Chubb et al., "Identification of two human dimethylarginine dimethylaminohydrolases with distinct tissue distributions and homology with microbial arginine deiminases," Biochemical Journal, vol. 343, no. 1, pp. 209-214, 1999.

[22] J. M. Sasser, N. C. Moningka, M. W. Cunningham Jr., B. Croker, and C. Baylis, "Asymmetric dimethylarginine in angiotensin IIinduced hypertension," American Journal of Physiology, vol. 298, no. 3, pp. R740-R746, 2010.

[23] M. F. Chen, X. M. Xie, T. L. Yang et al., "Role of asymmetric dimethylarginine in inflammatory reactions by angiotensin II," Journal of Vascular Research, vol. 44, no. 5, pp. 391-402, 2007.

[24] K. Sydow and T. Münzel, "ADMA and oxidative stress," Atherosclerosis Supplements, vol. 4, no. 4, pp. 41-51, 2003.

[25] J. F. Reckelhoff and J. C. Romero, "Role of oxidative stress in angiotensin-induced hypertension," American Journal of Physiology, vol. 284, no. 4, pp. R893-R912, 2003.

[26] C. Delles, M. P. Schneider, S. John, M. Gekle, and R. E. Schmieder, "Angiotensin converting enzyme inhibition and angiotensin II AT1-receptor blockade reduce the levels of asymmetrical NG, NG-dimethylarginine in human essential hypertension," American Journal of Hypertension, vol. 15, no. 7 , pp. 590-593, 2002.

[27] A. Ito, K. Egashira, T. Narishige, K. Muramatsu, and A. Takeshita, "Renin-angiotensin system is involved in the mechanism of increased serum asymmetric dimethylarginine in essential hypertension," Japanese Circulation Journal, vol. 65, no. 9, pp. 775-778, 2001.

[28] H. Wang and J. Liu, "Plasma asymmetric dimethylarginine and L-arginine levels in Chinese patients with essential hypertension without coronary artery disease," Journal of Cardiovascular Disease Research, vol. 2, no. 3, pp. 117-120, 2012. 


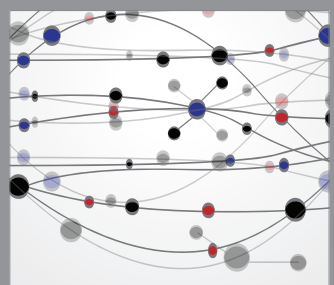

The Scientific World Journal
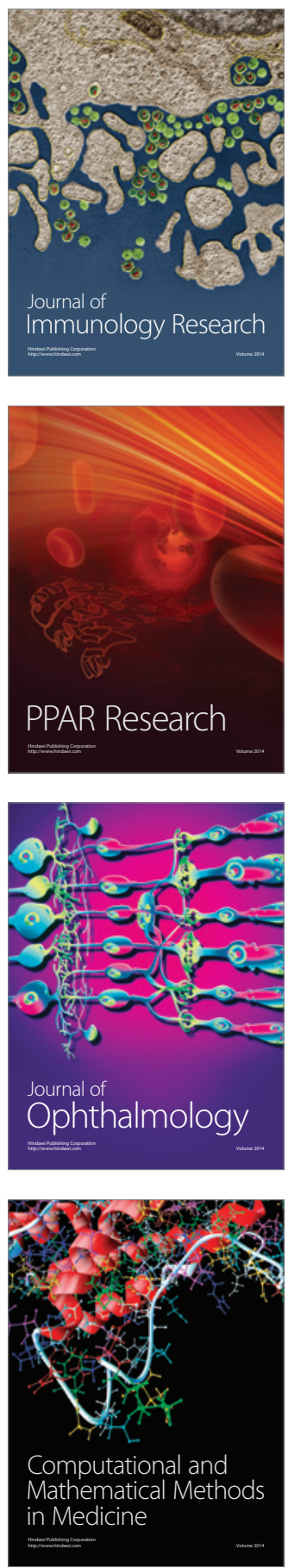

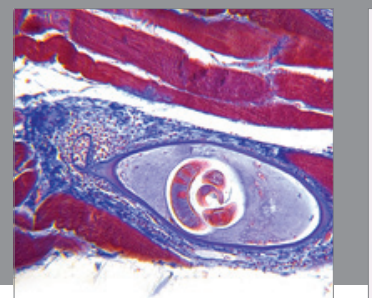

Gastroenterology

Research and Practice
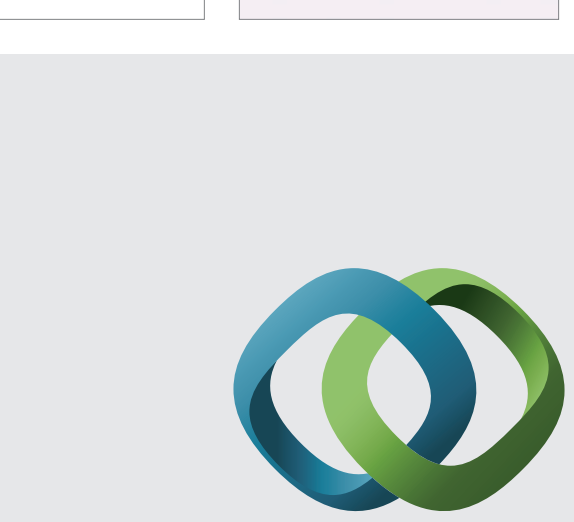

\section{Hindawi}

Submit your manuscripts at

http://www.hindawi.com
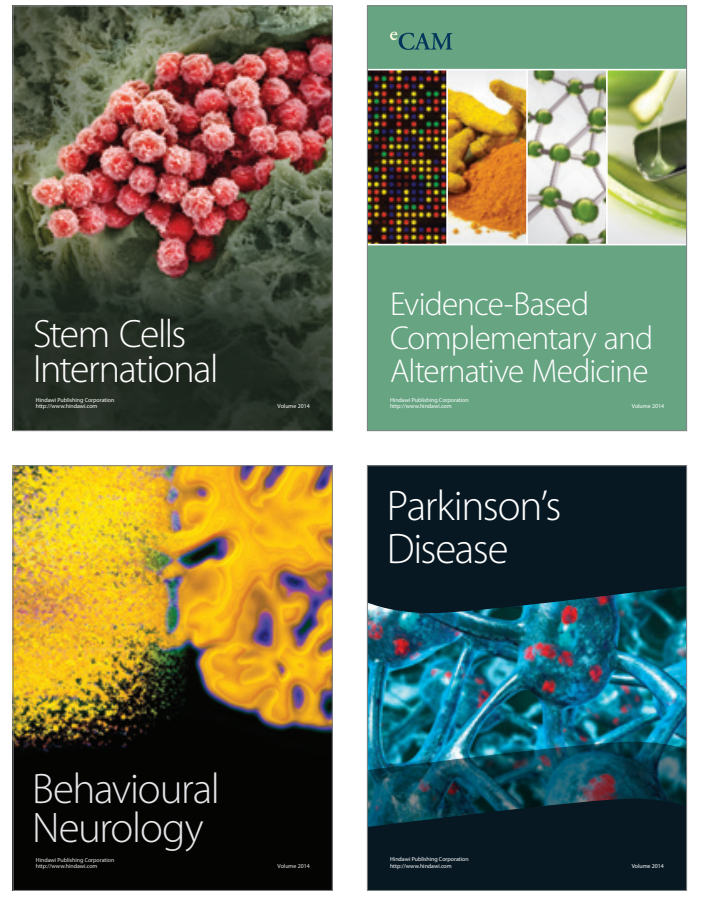
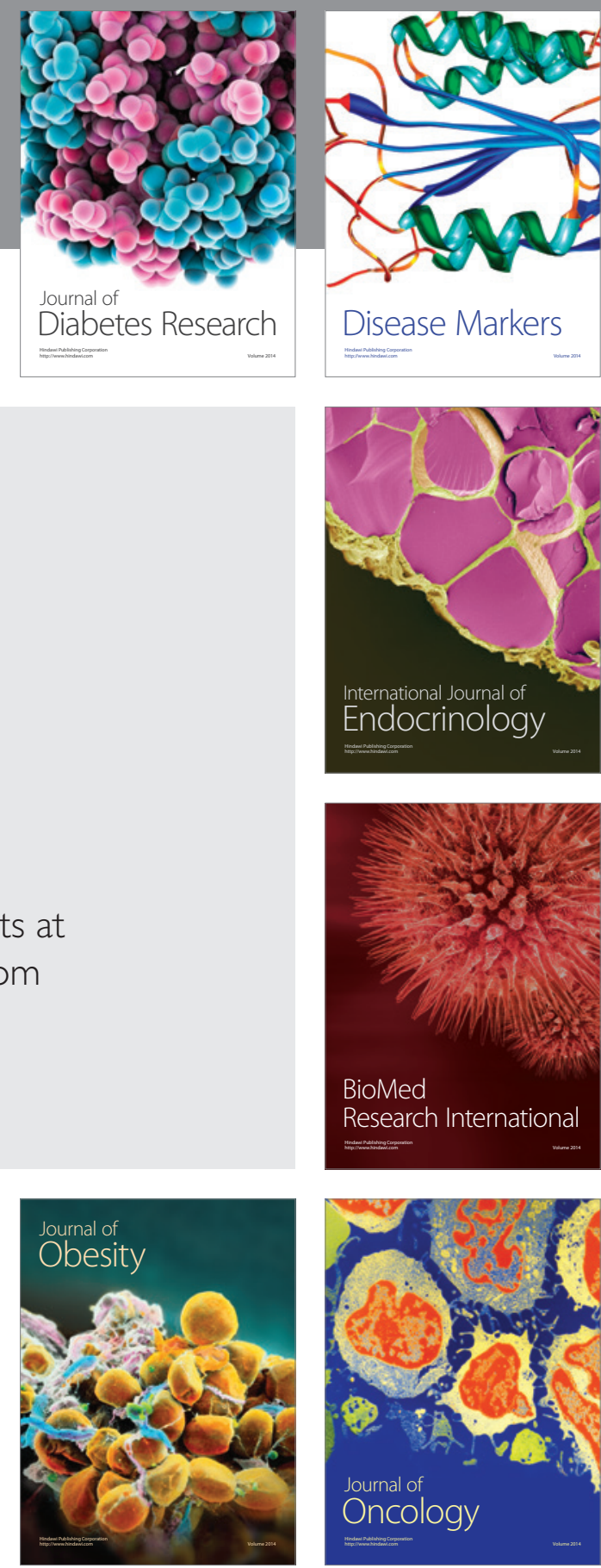

Disease Markers
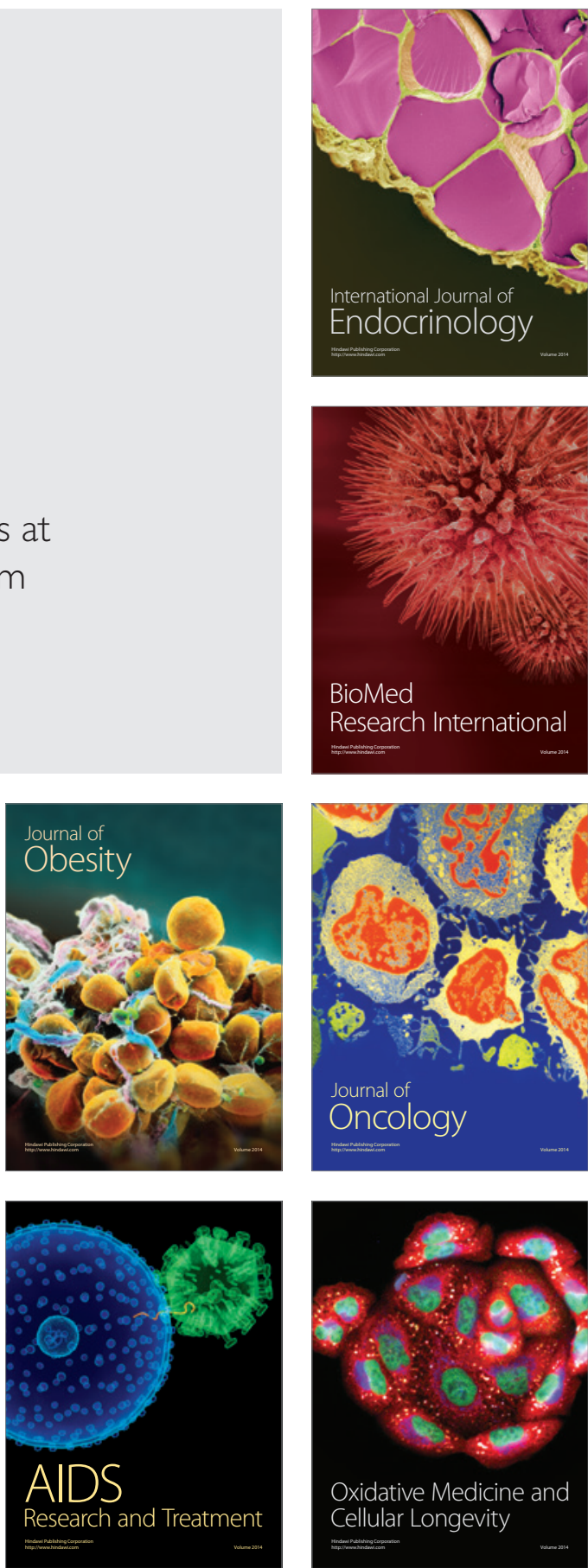